distinguishing normal capillaroscopic findings from (pathognomonic) abnormal (pathological) findings (scleroderma pattern), lies in the fact that this distinction allows the differentiation between a primary RP (not connected to any connective tissue disease [CTD]) from a secondary RP due to systemic sclerosis (SSc) and diseases of the scleroderma spectrum.

What is normal in primary RP?

A normal capillaroscopic pattern, by qualitative assessment, is characterized by a homogeneous distribution of hairpin shaped capillaries as a "comb-like structure", with a density of $>7$ capillaries per $\mathrm{mm}$, with a normal dimension and absence of large hemorrhages Yet, there exists a wide intra- and inter-individual variety in a normal population which will be discussed in this session.

What is pathognomonic abnormal in patients with RP due to SSc?

Patients with the RP who have an underlying clinically recognizable (= with skin involvement) SSc show a very characteristic combination of capillary abnormalities in the nailfold, which can easily be assessed through qualitative assessment (= pattern recognition). Maricq et al. described last century, with the widefield technique (magnification X12-14) the scleroderma pattern. This pathognomonic combination contains the following: a striking widening of all three segments of the capillary loop (arterial, venous and intermediate), loss of capillaries and disorganization of the nailfold capillary bed. Many branched "bushy" capillaries may also be observed.

In 2000, Cutolo et al. qualitatively assessed the nailfolds of an SSc cohort with patients fulfilling the American College of Rheumatology (ACR) criteria for SSc with the nailfold videocapillaroscopic (NVC) technique (magnification X200). According to the different proportions of the hallmark parameters of the scleroderma pattern (giants, capillary loss, hemorrhages and abnormal shapes: (neo) angiogenesis, Cutolo et al. defined three patterns "early", "active" and "late".

The central role of capillaroscopy in distinction between a primary and secondary RP due to SSc is reflected by the fact that capillaroscopy is one of the new ACR/ EULAR criteria for classifying a patient as having SSc.

In this lecture the standard "FAST TRACK" recognition system of the EULAR Study Group on Microcirculation in Rheumatic Diseases to discern scleroderma patterns from non-scleroderma patterns will be taught to the attendees.

Suggested further reading:

[1] Smith V, et al. Rheumatology (Oxford). 2016;55(5):883-90.

[2] Cutolo M, et al. Rheumatology (Oxford). 2018;57(4):757-759.

[3] Cutolo M, et al. Autoimmun Rev. 2018;17(4):344-352.

Disclosure of Interests: None declared

DOI: 10.1136/annrheumdis-2019-eular.8591

\section{SP0131 RELIABILITY IN THE LAST 10 YEARS: CAPILLAROSCOPIC CHARACTERISTICS VERSUS INSTRUMENTAL DETECTION}

Ariane Herrick. University of Manchester, Salford Royal Hospital NHS Foundation Trust, Division of Musculoskeletal and Dermatological Sciences, NIHR Manchester Musculoskeletal Biomedical Research Centre, Manchester, United Kingdom

Background: With nailfold capillaroscopy there are different aspects of reliability to consider, including reliability of qualitative image grading (e.g. 'early', 'active' and 'late') and also of semi-quantitative and quantitative measures including capillary density, apical width and presence of giant capillaries. The reliability of image acquisition (i.e. test-retest reliability) is especially important if nailfold capillaroscopic parameters are to be used in longitudinal studies (e.g. clinical trials) which involve acquiring repeat images over time. Lastly when assessing reliability, it is important to recognise that the nailfold capillaries cannot always be clearly seen, and cannot therefore be evaluated.

Objectives: To highlight the different aspects of reliability relating to nailfold capillaroscopy and how these have been addressed in studies over the last 10 years.

Methods: Review of recent studies.

Results:

1. Intra-observer reliability has been shown in several studies to be higher than inter-observer.

2. Assessment of 'evaluability' varies between observers and therefore also needs to be taken into account when assessing reliability.

3. Subject to evaluability, certain parameters demonstrate high intra- and inter-observer reliabilities. Reliability differs across different capillaroscopic parameters.

Conclusion: Recent studies examining reliability of capillaroscopy suggest that certain parameters, including image grade, capillary density and apex width have high intra-and inter-observer reliabilities (subject to nailfold image evaluability, which remains a major challenge). Standardised training is likely to improve reliability.

\section{REFERENCES}

[1] Murray AK, Vail A, Moore TL, et al. The influence of measurement location on reliability of quantitative videocapillaroscopy in patients with SSc. Rheumatol 2012; 51 : 1323-30.

[2] Overbury R, Murtaugh MA, Fisher A, et al. Primary care assessment of capillary abnormalities in patients with Raynaud's phenomenon. Clin Rheumatol 2015; 34: 2135-40.

[3] Smith V, Beeckman S, Herrick AL, et al. An EULAR study group pilot study on reliability of simple capillaroscopic definitions to describe capillary morphology in rheumatic diseases. Rheumatol 2016; 55: 883-90.

[4] Dinsdale G, Moore T, O'Leary N, et al. Intra-and inter-observer reliability of nailfold videocapillaroscopy - A possible outcome measure for systemic sclerosis-related microangiopathy. Microvascular Research 2017; 112: 1-6.

[5] Dinsdale G, Moore T, O'Leary N, et al. Quantitative outcome measures for systemic sclerosis-related microangiopathy - reliability of image acquisition in nailfold capillaroscopy. Microvascular Research 2017;113:56-9.

[6] Boulon C, Devos S, Mangin M, et al. Reproducibility of capillaroscopic classifications of systemic sclerosis: results from the SCLEROCAP study. Rheumatology 2017; 56: 1713-20.

[7] Cutolo M, Melsens K, Herrick AL, et al. Reliability of simple capillaroscopic definitions in describing capillary morphology in rheumatic diseases. Rheu matology (Letter) 2018; 57: 757-9.

Disclosure of Interests: None declared

DOI: 10.1136/annrheumdis-2019-eular.8499

\section{SP0132 METHODS AND TOOLS FOR QUANTIZATION OF CAPILLAROSCOPIC MORPHOLOGICAL CHANGES}

Francesca Ingegnoli. Academic Rheumatology Unit Universita degli Studi di

Torino, Academic Rheumatology Centre, Turin, Italy

Nailfold capillaroscopy is the one most used technique in both clinical and research settings by adult physicians and paediatric rheumatologists to assess patients with Raynaud's phenomenon as shown by an international survey on non-invasive techniques to assess the microcirculation performed under the aegis of members of the EULAR Study Group on Microcirculation in Rheumatic diseases [1].

Nailfold capillaroscopy is a simple non-invasive imaging technique mainly used to observe capillaries on the skin surface. After application of a drop of immersion oil, capillaries can be observed with a magnification lens because they run parallel to the epidermis at the nailbed area [2]

A number of different instruments can be used to perform the exam. They have different characteristics in terms of their cost, quality of images, magnifications, training period, portability, software for image analysis and storage.

Some of these instruments can be used both in clinical and research settings such as the stereomicroscope and the videocapillaroscope. The stereomicroscope allows the widefield visualization of the nailfold with low magnifications, the training is relatively short, but the examination is difficult to perform in patients with digital flexion contractures.

There appears to be consensus regarding the use of videocapillaroscopy that allows a detailed visualization of capillary morphology using higher magnifications (100-300x). Contact probe with polarized light microscopy permits easier observation of the skin surface, and the training period is briefer. Specific software are available for images analysis, storage, and complete medical reports (text + images) can be produced. By contrast, in a clinical setting, nailfold capillaries can generally be visualized using more simple, but also efficient tools such as a dermatoscope USB microscope, ophtamloscope or smartphone device. The quality of images can be quite good, although the lower magnification means that some details are unlikely to be seen, and they often lack the possibility of image storage and measurement. In particular, the dermatoscope with magnification of the order of $x 10$ is a small, inexpensive and easily portable piece of equipment that has been suggested to be comparable to videocapillaroscopy in routine clinical practice. As the study of capillary morphology provides clinically relevant information in the management of patients with scleroderma-spectrum diseases, the development of specific software to standardize and automatize the analysis is ongoing [3-4].

\section{REFERENCES:}

[1] Ingegnoli F, Smith V, Sulli A, Cutolo M. Capillaroscopy in Routine Diagnostics: Potentials and Limitations. Curr Rheumatol Rev. 2018 20;14(1):5-11

[2] Ingegnoli F, Ughi N, Dinsdale G, Orenti A, Boracchi $\mathrm{P}$, Allanore $\mathrm{Y}$, et al.; EULAR Study Group on Microcirculation in RheumaticDiseases. An international SUrvey on non-iNvaSive tecHniques to assess the mlcrocirculation in patients with RayNaud's phEnomenon (SUNSHINE survey). Rheumatol Int. 2017;37(11):1879-1890 